\title{
Physiotherapie beim Kreuzbandriss - Welche Maßnahmen sind sinnvoll?
}

\author{
Iris Challande-Kathmann
}

Der Kreuzbandriss (KBR) ist eine der häufigsten Erkrankungen an den Hintergliedmaßen des Hundes. Er kann konservativ oder chirurgisch behandelt werden. Bei beiden Methoden wird die Physiotherapie als Teil der Rehabilitation wärmstens empfohlen. Der Artikel behandelt zusätzlich zu Pathophysiologie, Klinik, Diagnose und den einzelnen Therapiemöglichkeiten die wichtigsten Aspekte der Physiotherapie und die speziellen Probleme, die in Abhängigkeit von den verschiedenen operativen Methoden bestehen.

\section{Physiologie}

Das gesunde Kniegelenk wird durch einen komplexen Bandapparat und die Muskulatur ( Abb.1) zusammengehalten. Die Flexion und Extension des Kniegelenks erfolgt in sagittaler Ebene. Das volle Bewegungsausmaß beträgt um die $140^{\circ}$.

\section{Ätiologie}

Mittelgroße bis große Rassen sowie kastrierte Tiere sind im Alter von 4-8 Jahren für einen Kreuzbandriss (KBR) prädisponiert. In ca. 30\% der Fälle sind beide Seiten mehr oder weniger stark betroffen.

Die Degeneration der Bänder ist Bestandteil des Alterns. Bei größeren Rassen treten Veränderungen wie ein Verlust der Faserorganisation und der Fibroblasten früher auf als bei kleinen Rassen. Verminderte Sexualhormone (Kastration) können den Kollagenmetabolismus negativ beeinflussen, sodass die Bänder dünner werden und weniger Elastin enthalten. Fehlstellungen der Tibia und Übergewicht begünstigen durch die Mehrbelastung ebenfalls einen Kreuzbandriss. In den meisten Fällen (95\%) ist die Ruptur Folge einer progressiven Degeneration der Bandfasern, sodass schon kleine repetitive „Traumata“ (wie Sprünge oder „stop and goes“) zum Reißen führen. Rein traumatisch bedingte Rupturen, die prinzipiell bei allen Rassen auftreten können, sind viel seltener als degenerativ bedingte $[2,9]$.

\section{Pathophysiologie}

Durch den Riss des vorderen Kreuzbands werden eine Innenrotation und eine Kranialbewegung der Tibia möglich (Subluxation). Infolge der Instabilität (repetitive Subluxation) entstehen meist Knorpeldegenerationen und ein Meniskusschaden. Es kommt zu einer Gelenkentzündung und damit verbundenen Schmerzen sowie zu einer Lahmheit. Das umliegende Gewebe schwillt an, insbesondere medial. Die umliegende Muskulatur reagiert zur Stabilisierung mit erhöhter Spannung. Besonders der M. quadriceps und der $\mathrm{M}$. gastrocnemius sind häufig schmerzhaft verspannt. Der Körper bildet infolgedessen verschiedene Mechanismen, um das Kniegelenk zu stabilisieren. Dabei kommt es zur Bildung von Osteophyten sowie zur Kapselverdickung. Die Gliedmaße wird unphysiologisch belastet. Das Kniegelenk wird aufgrund der schmerzhaften Extension im Gang und im Stand vermehrt in Flexion gehalten $[2,9]$.

\section{Klinik}

Im Gegensatz zum kaudalen Kreuzbandriss kommt die Ruptur des kranialen Kreuzbands beim Hund relativ häufig vor. Die Instabilität im Knie und die damit verbundene Entzündung führen zu einer hochgradigen Lahmheit. Nicht selten wird der mediale Meniskus beim kranialen Kreuzbandriss geschädigt, was zu einer weiteren Entzündung und zu
Schmerzen führt. Je nach Ausmaß der Meniskusschädigung kann es zu einem hörbaren Klicken kommen.

Bei einem partiellen Anriss ist die Lahmheit meist dezenter und die Erkrankung schwieriger zu diagnostizieren. Bei vielen Hunden ist die Lahmheit über Monate chronisch-intermittierend, bis es zu einem kompletten Riss und einer hochgradig akuten Lahmheit kommt.

Durch den Kreuzbandriss verändert sich die Gelenkbewegung im Sitzen und während des gesamten Gangzyklus. Während der Standphase subluxiert die Tibia abrupt nach kranial, um zu Beginn der Schwingphase wieder in die normale Position zurückzufallen. Die Tiere kompensieren dies durch eine verminderte Belastung der lateralen Gliedmaßenseite und eine vermehrte Flexion des Kniegelenks während des gesamten Gangzyklus. Sie laufen initial häufig auf 3 Beinen und setzen im Stand nur die Zehenspitzen auf. Später ist der Gang relativ

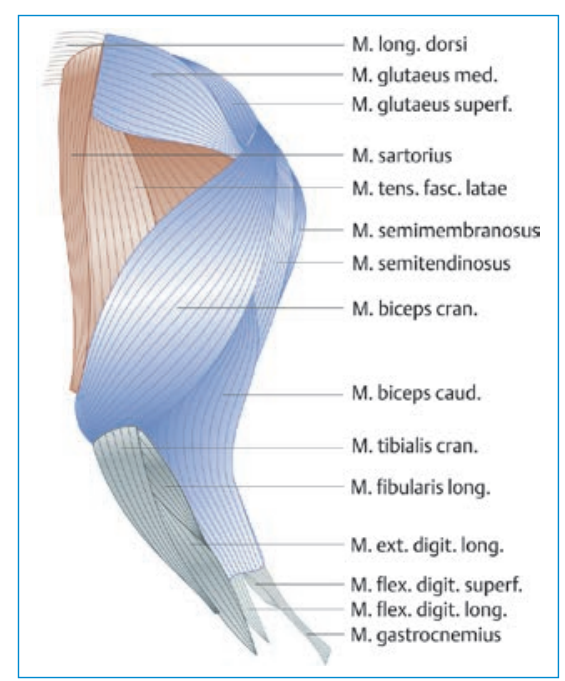

Abb. 1 Muskulatur des Kniegelenks (aus: Mai S. Physiotherapie und Bewegungstraining für Hunde. Sonntag Verlag, 2011). 
typisch mit einer Hyperflexion des Kniegelenks, die sich während der Standphase fließend verstärkt und wieder abnimmt. Das Gangbild kann somit entfernt an einen Breakdance-Schritt erinnern. Die Tiere rotieren im Sitzen die betroffene Gliedmaßen nach außen, um das Knie zu entlasten $(\triangleright$ Abb. 2) $[2,9]$.

\section{Diagnose}

Die Lahmheit und die Dolenz im Knie mit mehr oder weniger starker Umfangsvermehrung sind relativ typische Symptome. Ein positiver Schubladen- oder Tibiakompressionstest kann den Verdacht bestätigen. Allerdings sind diese auch bei einer kompletten Ruptur nicht immer einfach auszulösen. Gerade bei sehr großen und gut bemuskelten Hunden, in chronischen Fällen und bei partiellen Rissen kann die Durchführung erschwert sein. In Narkose wird hingegen aufgrund der verminderten Muskelspannung eher eine im Vergleich zur Gegenseite vorliegende Instabilität des Kniegelenks aufgedeckt.

Radiologisch werden in chronischen Fällen typische Anzeichen einer Arthrose gefunden. Der Tibiakompressionstest kann unter radiologischer Kontrolle auch partielle Risse aufdecken. Für eine Läsion des kranialen Kreuzbands ist dabei die Verschiebung des poplitealen Sesambeins nach distal typisch. Auch eine Magnetresonanztomografie kann bei der Diagnosestellung zum Einsatz kommen. Die mögliche Evaluation des Meniskus bietet dabei einen Vorteil [2,9].

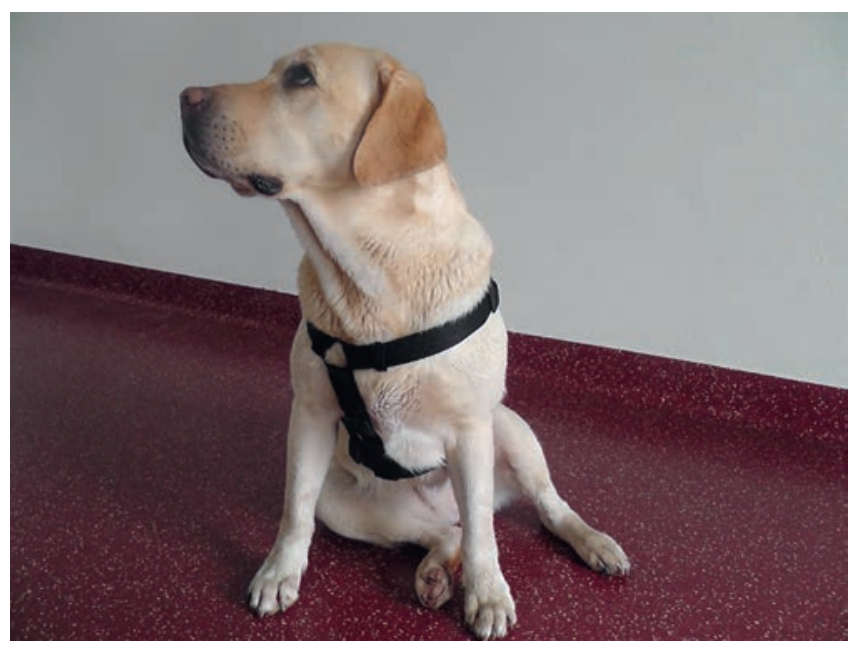

Abb. 2 Das linke Knie wird zur Entlastung im Sitzen nach lateral positioniert.
- Kontrakturen der Oberschenkelmuskulatur (mit z.T. starkem Zug auf das Hüftgelenk) und der kaudalen Unterschenkelmuskulatur

- fortschreitende Knorpeldegeneration

- Gelenkkapselkontraktur

- eingeschränkte Beweglichkeit des Kniegelenks

- schmerzhafte Verspannungen der gesamte Wirbelsäulenmuskulatur infolge der Fehlbelastung

\section{Konservative Therapie}

Je nach Alter, gesundheitlichem Zustand des Tieres und der Überzeugung oder finanziellen Situation des Besitzers ist ein konservatives Vorgehen anzuraten. Auch bei kleineren Hunden, die weniger als 15-20 kg wiegen, kann ein konservativer Therapieversuch unternommen werden.

Persistiert die Lahmheit bei einem Kreuzbandriss über 6-8 Wochen, ist ein größerer Meniskusschaden wahrscheinlich und ein operativer Eingriff anzuraten.

Die Tiere stehen mindestens 4 Wochen unter striktem Leinenzwang. Abrupte Bewegungen sind zu vermeiden. Insbesondere das unkontrollierte Herunterlaufen der Treppe oder das Herunterspringen sind schmerzhaft, sodass die Tiere wieder eine vermehrte Lahmheit zeigen. Nach Bedarf sollte das Körpergewicht reduziert werden und Entzündungshemmer sollten - solange wie nötig - zum Einsatz kommen. Die Erfolgsaussichten sind bei adäquater Physiotherapie auch bei größeren Hunden und insbesondere bei partiellem Kreuzbandriss günstig. Bei übergewichtigen Hunden zeigte sich in einer Studie innerhalb 1 Jahres eine Erfolgsrate von 66\% [10].

Eine genügende Kniestabilität kann nicht nur durch eine Operation, sondern auch durch eine neuromuskuläre Rehabilitation erreicht werden [3]. In der Humanmedizin belegen immer mehr wissenschaftliche Studien, dass eine konservative Therapie bei vielen Patienten zufrieden stellende Resultate bringt. Die intensive und frühzeitige physiotherapeutische Behandlung ist dabei ein fester Bestandteil der Therapie. Die Studien be- 
legen zudem, dass eine chirurgische Therapie keine Garantie für die Wiederaufnahme der sportlichen Aktivitäten darstellt. In der Veterinärmedizin wird hingegen von vielen Tierärzten immer noch die Meinung vertreten, dass ein Kreuzbandriss schnellstmöglich operiert werden muss. Es gibt jedoch keine wissenschaftlichen Studien, die dies belegen. Die Fortschritte, die in den letzten Jahren in der Veterinärphysiotherapie gemacht wurden, werden noch immer unterschätzt. Es werden daher weitere wissenschaftliche Studien benötigt, die aufzeigen, dass eine konservative Therapie beim Hund ebenso gute Erfolge bringen kann wie in der Humanmedizin.

Das Rehabilitationsprogramm der konservativen Therapie ähnelt dem Vorgehen, das bei einer chirurgischen Stabilisierung des Kreuzbandrisses beschrieben wird.

\section{Präoperative Physiotherapie}

Da in den meisten Fällen ein chronischer Verlauf typisch ist, kann bei geplanter Operation bereits vor dem Eingriff mit der Physiotherapie begonnen werden. Dies ist von Vorteil, da die postoperative Regeneration verkürzt wird und dem Tier die Übungen schon vor dem Eingriff bekannt sind, sodass der Stress beim frisch operierten Hund vermindert werden kann. Außerdem sprechen die Hunde z.T. so gut auf die Physiotherapie an, dass eine Operation vermieden werden kann.

Jedes Tier erhält ein individuelles Programm, das an den Krankheitsverlauf, die Therapiemethode und die jeweiligen Bedürfnisse angepasst ist [2]. Dabei stehen der Muskelaufbau auf dem Unterwasserlaufband, Massagen, Dehnungen und passive Bewegungen des Kniegelenks - so wie sie bei der chirurgischen Therapie beschrieben werden - im Vordergrund.

\section{Chirurgische Therapie}

Es wurden über 50 verschiedene Operationstechniken beschrieben. Diese werden unterteilt in:

- extrakapsuläre und intraartikuläre Stabilisation
- „tibia plateau leveling osteopathie“ (TPLO)

- „tibial tuberosity advancement“ (TTA)

Leider gibt es zurzeit keine chirurgische Methode, bei der ausreichend Daten belegen, dass die Tiere regelmäßig mit guten Aussichten zu einer normalen Gelenkfunktion zurückkehren $[1,2,9]$.

Zusätzlich zu der Stabilisierung ist die Reinigung des Gelenks beim operativen Eingriff wichtig. Dabei werden Bandreste entfernt sowie Knorpel- und Meniskusschäden abgetragen. Der gemeinsame Nenner aller Methoden scheint eine beschleunigte Kapselfibrose mit Stabilisierung auf Kosten des Bewegungsausmaßes des Gelenks zu sein. Ausnahmen sind die TPLO und die TTA-Techniken, bei denen die Wirksamkeit nicht auf dem Ersatz des Kreuzbands beruht. Das Kreuzband ist durch die veränderte Statik des Gelenks nicht mehr so wichtig für die Funktion des Kniegelenks.

\section{Extrakapsuläre Stabilisierungen}

Bis auf die Methode der Fibulakopftransposition sind extrakapsuläre Stabilisierungen leichter und schneller durchführbar als intraartikuläre. Der klinische Erfolg liegt bei ca. 90\%, obwohl die Arthrose weiter voranschreitet und nach ein paar Monaten meist die vordere Schublade wieder in unterschiedlichem Ausmaß ausgelöst werden kann. Die über den Zeitraum von 8-10 Wochen entstehende Kapselfibrose ist für die Stabilisierung des Gelenks verantwortlich. Rupturen des hinteren Kreuzbands werden meist durch extrakapsuläre Techniken stabilisiert.

\section{Intraartikuläre Stabilisierung}

Die Methode wird kaum noch durchgeführt. Das Kreuzband wird bei dem Eingriff entweder durch biologisches (z.B. Faszie) oder synthetisches Material ersetzt. Das biologische Material wird zwischen der 2. und 20. Woche schwächer und kann überdehnt werden oder reißen, bis es nach erfolgter Revaskularisierung und Integration seine volle Widerstandsfähigkeit gewinnt. Dies muss bei der physiotherapeutischen Therapie berücksichtigt werden.

\section{Kreuzbandriss bei der Katze}

Rupturen des vorderen Kreuzbands sind bei Katzen deutlich seltener als bei Hunden. Zugrunde liegen die gleichen Pathomechanismen wie beim Hund: die traumatisch und die progressiv degenerativ bedingte Ruptur. Auch bei der Katze spielen Faktoren wie Alter, Körpergewicht und körperliche Aktivität eine wichtige Rolle. Dabei treten rein traumatisch bedingte Rupturen des Kreuzbands häufiger auf als degenerative.

Bei Katzen ist die konservative Therapie in den meisten Fällen erfolgreich. Wenn weitere Verletzungen der Bänder vorliegen, die Instabilität zu groß ist und die Katze nach einer etwa 2-wöchigen konservativen Therapie das Bein noch immer nicht belastet, wird meist extrakapsulär stabilisiert [2, 9].

Ein Verband wäre bei der intraartikulären und extrakapsulären Stabilisierung zur Minderbelastung der Implantate über einen längeren Zeitraum wünschenswert. Die Nachteile der Immobilisation überwiegen aber [2, 7,9]:

- Muskelatrophie

- Knorpeldegeneration

- intraartikuläre Narbenbildung

\section{TPLO}

Die Operation basiert auf dem Prinzip, die Subluxation der Tibia nach kranial während der Standphase zu vermeiden. Dies erfolgt durch eine Kombination der veränderten Biomechanik des Gelenks und einer aktiven Muskelkontraktion. Dabei kommt der kaudale Teil nach der Osteotomie durch Rotation des Tibiaplateaus mehr proximal zum Liegen. Er wird angehoben, sodass das Kaudalgleiten des Femurs im Stand vermindert werden kann. Die periartikuläre Muskulatur trägt zusätzlich zur Kniestabilität bei.

Die postoperativen Komplikationen beruhen auf der veränderten Biomechanik im Gelenk. Insbesondere Patellarbandentzündungen (Desmitis) sind häufig im 1. postoperativen Monat zu beobachten. 
Das Band ist lockerer und schmerzt bei Palpation, insbesondere an seiner Insertionsstelle an der Crista tibiae. Weitere Komplikationen sind ein Teilabriss der Crista tibiae oder eine Verletzung des medialen Meniskus. Diese sind meist selbstlimitierend durch Ruhe, Analgetika und Kältetherapie.

\section{TTA}

Die Biomechanik des Kniegelenks wird wie bei der TPLO verändert, sodass die Scherkräfte in der Extension verringert werden und das vordere Kreuzband nicht mehr zur Stabilisation benötigt wird. Im Gegensatz zur TPLO kommt es aber zu einer Druckverminderung zwischen Patella und Femur. Dies führt bei Knorpelschäden im Femoropatellargelenk sofort zu einer Schmerzminderung.

Wie bei der TPLO kann es durch die geänderte Statik postoperativ jedoch zu einem Schaden des medialen Meniskus kommen $[2,4]$.

\section{Postoperative Physiotherapie}

Die Physiotherapie sollte einen festen Bestandteil des postoperativen Managements bilden $[2,6,8]$.

Für die Rehabilitation des hinteren Kreuzbands gelten dabei die gleichen Prinzipien wie für das vordere Kreuzband.

\section{Kältetherapie}

In den ersten 2 Stunden postoperativ wird für etwa 15 Minuten ein Coldpack auf das Knie gelegt. Die Kühlung kann mehrmals im Abstand von 2 Stunden wiederholt werden. Auf diese Weise werden Entzündungen, Schwellungen und Schmerzen vermindert. Bei Bedarf kann nach den Übungen ebenfalls Kälte aufgetragen werden.

\section{Wärmetherapie}

Nach ca. 1 Woche kann - sofern das Gelenk nicht vermehrt warm ist - vorbereitend auf die passive Bewegungstherapie für 15 Minuten ein Hotpack aufgelegt werden. Dies erhöht die Elastizität der fibrösen Strukturen und vermindert die Viskosität der Synovia, die so für die Knorpelernährung besser im Gelenk verteilt wird. Rotlicht oder therapeutischer Ultraschall können bei starken Verspannungen und Kontrakturen Anwendung finden und zur Vorbereitung der Muskulatur auf Dehnungen und Massagen eingesetzt werden.

\section{Passive Bewegungstherapie}

Das Kniegelenk wird ab dem 1. postoperativen Tag bzw. nach Entfernen des Verbands vorsichtig 3-mal täglich mindestens 10-mal passiv in alle physiologischen Richtungen bewegt $(\checkmark$ Abb.3). Die Muskulatur (v.a. M. quadriceps und M. gastrocnemius) wird gedehnt ( $\bullet$ Abb.4). So bleibt die Beweglichkeit erhalten oder kann wiederhergestellt werden. Kontrakturen der Muskulatur und der Gelenkkapsel sowie Knorpeldegenerationen können verhindert werden. Hüfte und Tarsus sollten aufgrund der Muskelspannungen ebenfalls täglich passiv bewegt werden.

\section{Massage}

Die Massage steigert die Durchblutung (Sauerstoff- und Nährstoffantransport Schlackenabtransport), vermindert Schmerzen, löst Verspannungen und mit der Zeit auch Kontrakturen. Die Patella wird vorsichtig mobilisiert. Ab dem 1. postoperativen Tag kann 1-mal wöchentlich oder nach Bedarf eine Ganzkörpermassage durchgeführt werden, um die durch die Fehlbelastung entstandenen Verspannungen der Gegenseite, des Rückens sowie der Vordergliedmaßen zu lösen.

\section{Hydrotherapie}

Die Gangschulung auf dem Unterwasserlaufband kann bei guter Wundheilung nach Entfernen der Fäden durchgeführt werden. Idealerweise sollte das Tier dabei wöchentlich 1- bis 3-mal langsam aufbauend trainiert werden. Das Training wird individuell an die Kondition des Tieres angepasst. Meist ist eine progressive Steigerung der Dauer von $5 \mathrm{Mi}$ nuten pro Training möglich. Dies erlaubt einen guten Muskelaufbau und eine Steigerung der Gelenkbeweglichkeit mit gleichzeitig möglichst geringer Belastung der Gelenke und der Implantate. Das Tier sollte immer mindestens bis zur Mitte des Oberschenkels im Wasser stehen. Der Besitzer kann mit dem Patienten auch schwimmen gehen, um Kosten zu sparen.

\section{Aktive Bewegungstherapie}

Zu Beginn sind mindestens 5-mal täglich sehr langsame und kurze Spaziergänge (maximal 5 Minuten) an der Leine angezeigt. Nach ca. 2 Wochen können die
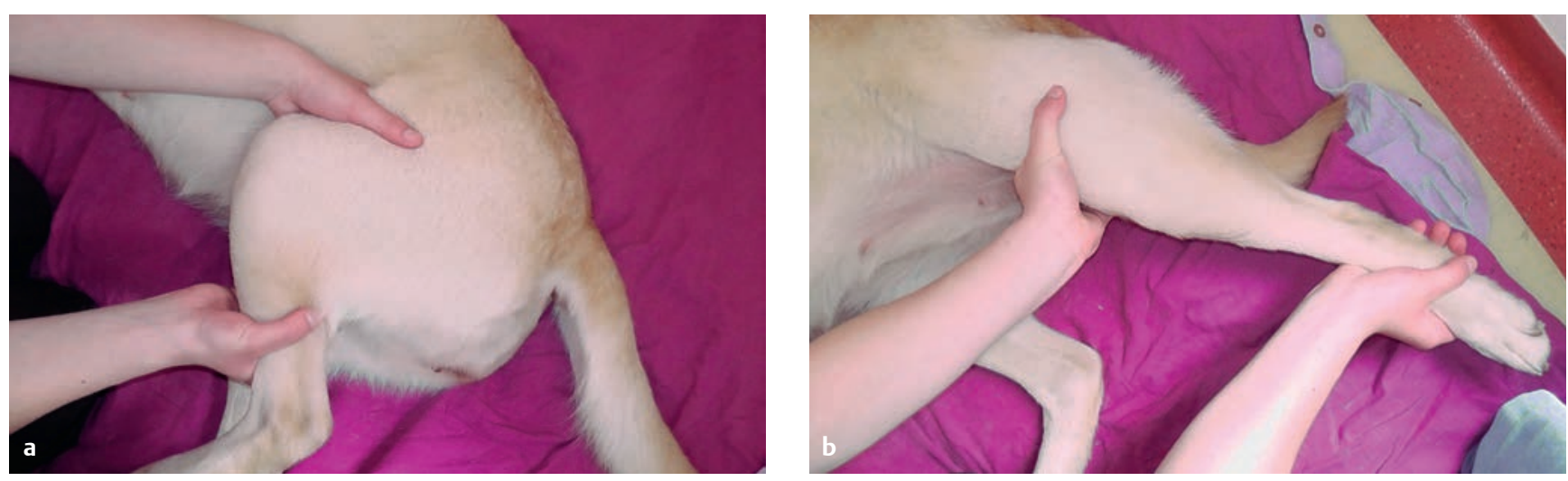

Abb. 3 Passive Bewegung des Kniegelenks. a Flexion. b Extension. 
Spaziergänge langsam über 1 Monat bis auf 15-20 Minuten gesteigert werden. $\mathrm{Zu}$ diesem Zeitpunkt werden zudem folgende Übungen in das Programm integriert:

- Gleichgewichtsübungen

- Sitz- und Stehübungen

- Gehen in Kreisen

- bergauf gehen

- im tiefen Untergrund gehen

- Hindernisarbeit ( Abb.5)

Für einen gleichmäßigen Muskelaufbau werden nach 2 Monaten Trabstrecken in das Programm eingebaut. Das Traben sollte kontrolliert an der Leine erfolgen.

\section{Physikalische Therapie}

Die Magnetfeld- und die Elektrotherapie (TENS lokal oder segmental [L3 - S1]) stellen gute zusätzliche Methoden zur Förderung der Heilung und zur Analgesie dar. Sie können je nach Verfügbarkeit 1- bis 2-mal täglich - je nach Herstellerangaben durchgeführt werden.

\section{Besonderheiten}

Im Folgenden werden therapeutische Besonderheiten beschrieben, die bei den unterschiedlichen Operationsmethoden Beachtung finden sollten.

\section{Extrakapsuläre Stabilisierungen}

In den meisten Fällen ist das Kniegelenk bereits direkt postoperativ relativ stabil, sodass sofort mit der Physiotherapie begonnen werden kann. Schwellungen und Entzündungen kann mit einer Kältetherapie entgegengewirkt werden. Die Schmerzen sind zusätzlich mit Analgetika unter Kontrolle zu halten. Die handelsüblichen nicht-steroidalen Entzündungshemmer sind in der empfohlenen Dosierung in der Regel ausreichend.

Bereits innerhalb der ersten 24 Stunden sollte das Tier maximal 5 Minuten mindestens 5-mal am Tag kontrolliert an der Leine geführt und dazu ermutiget werden, die Gliedmaße zu benutzen. Das Gelenk kann bereits am 1. Tag vorsichtig passiv bewegt werden. Dabei wird besonderen Wert auf die Extension des

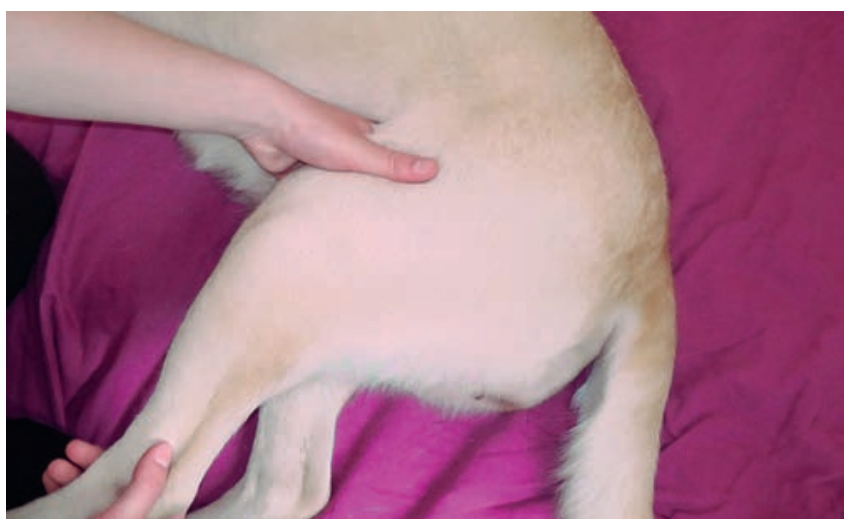

Abb. 4 Vorsichtiges Dehnen des M. quadriceps. 


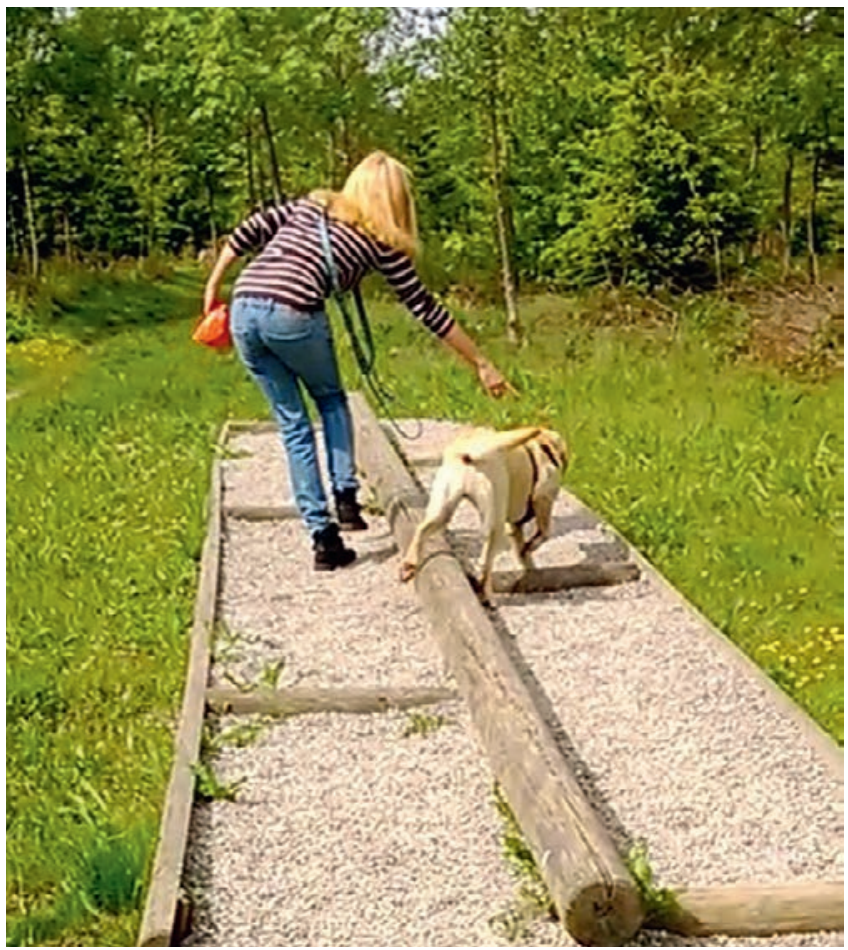

Abb. 5 Aktive Bewegungstherapie: Hindernislaufen in der späteren Phase der Rehabilitation.

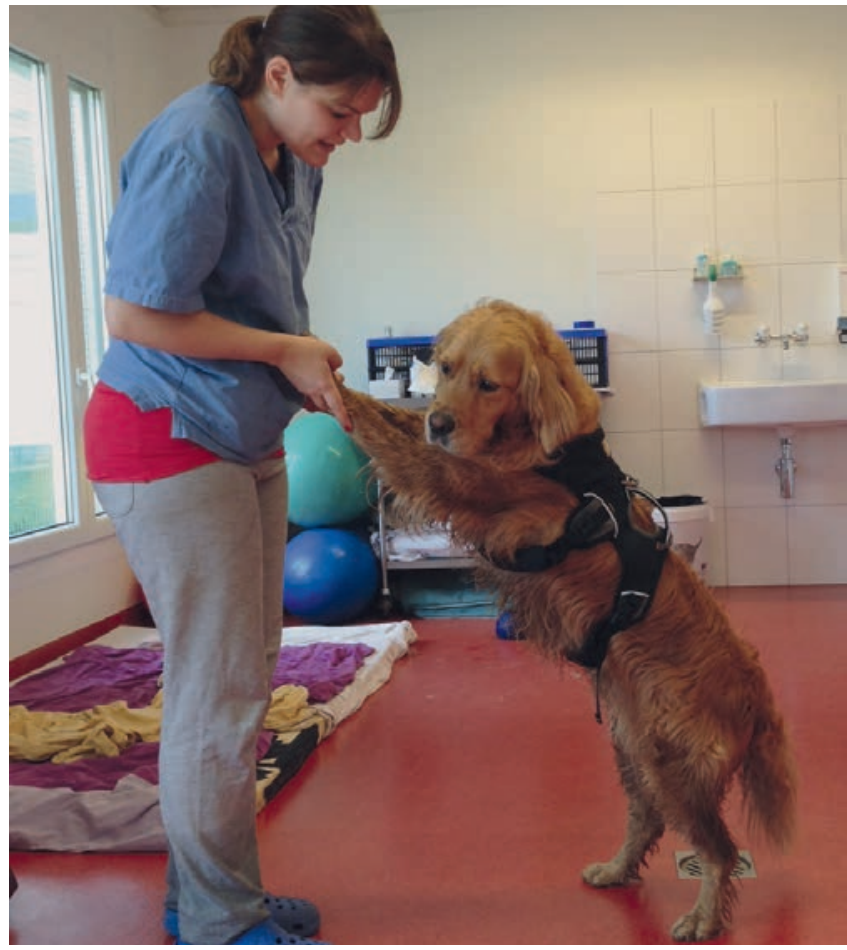

Abb. 6 Beim Tanzen muss der Hund auf den Hinterbeinen vor- und zurücklaufen.
Kniegelenks gelegt. Nach ca. 2 Wochen sollte das Ausmaß der Extension dem präoperativen Zustand gleichen.

Eine aktive Bewegungstherapie zur Stärkung der Nachhand kommt ebenfalls in den ersten Tagen zum Einsatz. Alle Bewegungen müssen dabei kontrolliert durchgeführt werden. Die ersten 2-3 Monate postoperativ ist das Tier ruhig zu halten. Abrupte Bewegungen oder Sprünge sind in dieser Phase nicht erlaubt.

\section{Intraartikuläre Stabilisierung}

Die Behandlungsmethoden und ihre Ziele sind die gleichen wie bei der extrakapsulären Stabilisierung. Die mögliche Implantatschwäche in den ersten Wochen muss mit dem Chirurgen besprochen und bei der aktiven Therapie berücksichtigt werden.

\section{TPLO}

Die Kältetherapie kann sofort begonnen werden.

Es muss darauf geachtet werden, dass das Coldpack nicht auf die medial gelegene Platte platziert wird.
Postoperativ kann in den ersten Tagen zudem mit passiver Bewegung und kontrolliertem Leinengang (nur ein paar Minuten) mit Unterstützung begonnen werden. Dabei ist für 4-6 Wochen eine starke Flexion des Knies während der Standphase zu vermeiden, um das Patellarband und die Crista tibiae nicht zu überlasten. Insbesondere Sprünge, Rennen und Treppensteigen sind mit einer starken Belastung verbunden und somit verboten. Die Physiotherapie verbessert die Kniegelenksflexion und -extension sowie den Aufbau der Muskulatur. Die Tiere sind früher lahmheitsfrei als ohne physiotherapeutische Behandlung $[2,5,8]$.

Bis zu 1 Monat nach der TPLO sollte die Flexion unter angepasster Hüftflexion durchgeführt werden, um das Patellarband zu schonen. Das Patellarband sollte nicht zu sehr unter Zug gesetzt werden. Daher wird empfohlen, vor dem Dehnen den M. quadriceps gut vorzuwärmen und v.a. mit Massagen zu arbeiten. Die betroffene Hintergliedmaße wird 3-mal täglich massiert.

Nach 4-6 Wochen (Heilungszeit für die Osteotomiestelle) kann die Belastung langsam gesteigert werden. Aktive Bewegungsübungen dürfen erst 5-6 Wo- chen nach dem operativen Eingriff in das Programm integriert werden und müssen sehr vorsichtig erfolgen. Laufen im tiefen Untergrund, Sitz- und Stehübungen sowie Tanzen ( $\bullet$ Abb.6) sind beispielsweise zu vermeiden. Nach 3 Monaten können für den gleichmäßigen Muskelaufbau Trabstrecken (kontrolliert an der Leine) in das Training integriert werden.

\section{TTA}

Die Physiotherapie sollte in den ersten Tagen begonnen werden. Die Prinzipien sind die gleichen wie bei der TPLO. In den ersten 8 Wochen werden kontrollierte Bewegungen an der Leine und dann eine progressive Rückkehr zur normalen Belastung empfohlen. Meist wird das Bein schon nach wenigen Tagen wieder belastet. Nach 4 Monaten sind die Tiere in der Regel lahmheitsfrei.

\section{Weiteres Prozedere}

Im Folgenden wird das weitere Prozedere beschrieben, wobei Abweichungen aufgrund des individuellen Vorgehens möglich sind.

In der Rehaklinik der Autorin werden die Hunde nach der Erstvorstellung und physiotherapeutischen Behandlung meist 


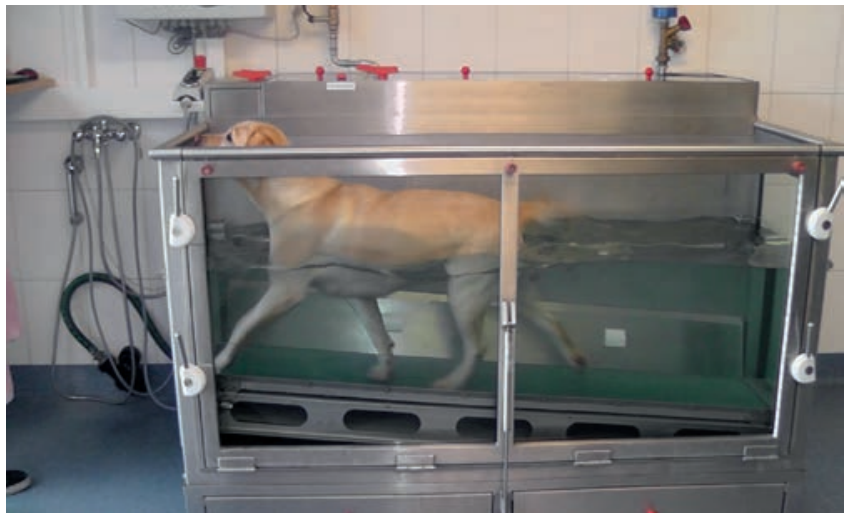

Abb. 7

Arbeit auf dem Unterwasserlaufband. Im Verlauf der Rehabilitation kann zur Förderung der Extension eine Steigung der Rampe integriert werden.

innerhalb der nächsten 2 Wochen 2-mal wöchentlich auf dem Unterwasserlaufband trainiert. Den Besitzern werden zudem Übungen für zu Hause gezeigt. Der Besitzer sollte dann nach genauer Instruktion 3-mal täglich die passiven Bewegungen und die Massage sowie 5-mal täglich die aktive Bewegungstherapie durchführen.

Eine regelmäßige Konsultation beim Physiotherapeuten (1- bis 3-mal/Woche in den ersten 2-3 Wochen) zur Kontrolle und Anpassung des Programms sowie für die Hydrotherapie auf dem Unterwasserlaufband ist zu empfehlen.

Manchmal werden die Tiere jedoch stationär zur intensiven Physiotherapie aufgenommen, da die Besitzer das Tier kaum ruhig halten können oder wenig Zeit haben. Dann werden sie neben der Durchführung der oben beschriebenen aktiven und passiven Bewegungstherapie täglich auf dem Unterwasserlaufband trainiert ( $\bullet$ Abb. 7) [2].

Nach den ersten 2 Wochen folgt neben der Hydrotherapie eine 2. Behandlung durch den Physiotherapeuten. Das Trai- ning auf dem Unterwasserlaufband setzt sich mit 1 Therapieeinheit pro Woche fort. Sofern keine Probleme auftreten, wird der Hund nach 1 Monat wieder vorgestellt. Danach wird das Training meist zu Hause an Land oder im Wasser (Schwimmen) fortgeführt oder es wird noch weiter auf dem Unterwasserlaufband trainiert.

Nach spätestens weiteren 2 Monaten kommen die Tiere erneut zur Kontrolle. Die Reha ist dann in der Regel abgeschlossen. Die Autorin hat mit zusätzlichen osteopathischen Behandlungen und/oder Akupunktur insbesondere bei konservativ behandelten Tieren sehr gute Erfahrungen gemacht.

\section{Online zu finden unter}

http://dx.doi.org/10.1055/s-0034-1384413

\section{Literatur}

1 Aragon CL, Budsberg SC. Applications of evidence-based medicine: cranial cruciate ligament injury repair in the dog. Vet Surg 2005; 34 (2): 93-98

2 Challande-Kathmann I. Rehabilitation und Physiotherapie bei Hund und Katze. Hannover: Schlütersche; 2009
3 Delincé P, Ghafil D. Anterior cruciate ligament tears: conservative or surgical treatment? Knee Surg Sports Traumatol Arthrosc 2012; 20: 48-61

4 Hoffmann DE, Miller JM, Ober CP et al. Tibial tuberosity advancement in 65 canine stifles. Vet Comp Orthop Traumatol 2006; 19 (4): 219-227

5 Jandi AS, Schulman AJ. Incidence of motion loss of the stifle joint in dogs with naturally occurring cranial cruciate ligament rupture surgically treated with tibial plateau leveling osteotomy: longitudinal clinical study of 412 cases. Vet Surg 2007; 36 (2): 114-121

6 Marsolais GS, Dvorak G, Conzemius MG. Effects of postoperative rehabilitation on limb function after cranial cruciate ligament repair in dogs. J Am Vet Med Assoc 2002; 220 (9): 1325-1330

7 Millis DL, Levine D, Taylor RA. Canine Rehabilitation and Physical Therapy. 2nd ed. Philadelphia: WB Saunders; 2014

8 Monk ML, Preston CA, McGowan CM. Effects of early intensive postoperative physiotherapy on limb function after tibial plateau leveling osteotomy in dogs with deficiency of the cranial cruciate ligament. Am J Vet Res 2006; 67 (3): 529-536

9 Slatter D. Textbook of Small Animal Surgery. 3rd ed. Philadelphia: Saunders, Elsevier; 2002

10 Wucherer KL, Conzemius MG, Evans R et al. Short-term and long-term outcomes for overweight dogs with cranial cruciate ligament rupture treated surgically or nonsurgically. J Am Vet Med Assoc 2013; 242 (10): 1364-1372

Dr. med. vet. Iris Challande-Kathmann, DECVN Zusatzbezeichnung

Physikalische Therapie/Physiotherapie Tierosteopathie dipl. IMAOV, Tierakupunktur dipl. IMAOV

INDIKA-animalreha SA

Route de la Rougève 74

1623 Semsales, Schweiz

info@indika.ch,www.indika.ch 